\title{
Ruled 4-manifolds and isotopies of symplectic surfaces
}

\author{
R. Hind ${ }^{*} \quad$ A. Ivrii
}

July 21, 2018

\begin{abstract}
We study symplectic surfaces in ruled symplectic 4-manifolds which are disjoint from a given symplectic section. As a consequence, in any symplectic 4-manifold, two homologous symplectic surfaces which are $C^{0}$ close must be Hamiltonian isotopic.
\end{abstract}

\section{Introduction}

This paper's initial objective was to address a question of local isotopy of symplectic surfaces in symplectic 4-manifolds (and this was originally motivated by the question of local isotopy of lagrangian surfaces in 4manifolds [6]). More precisely, let $(M, \omega)$ be a symplectic 4 -manifold and let $\Sigma \subset M$ be a compact embedded symplectic surface. It is natural to ask whether other symplectic surfaces in some neighborhood of $\Sigma$ are smoothly or symplectically isotopic to $\Sigma$ (that is isotopic through a family of smooth or symplectic embedded surfaces). In fact we will show (see corollary 8.3 the following:

Theorem 1.1. The set of symplectic surfaces homologous to $\Sigma$ and lying in a standard symplectic neighborhood of $\Sigma$ is (weakly) contractible.

In section 2 we will briefly describe what these standard symplectic neighborhoods (standard symplectic disk bundles over $\Sigma$ ) are, and refer the reader to [2] for a more complete description of this. We point out that any symplectic isotopy $\Sigma_{t}, t \in[0,1]$, is given by a Hamiltonian whose support at each $t$ is arbitrarily close to $\Sigma_{t}$ (a proof of this can be found in, for example, [14). We would like to add that in general homologous symplectic surfaces inside a symplectic 4-manifold do not need to be isotopic (see [4), however there are also cases when this is true (13, 14]).

This paper's real objective is to investigate the set of symplectic surfaces in ruled symplectic 4-manifolds. A 4-manifold $\bar{X}$ is called ruled if it is an $S^{2}$-fibration over a Riemann surface $\Sigma$, such symplectic manifolds have been extensively studied in the literature (see, for example, LalondeMcDuff [8, 9], and also 1], 11). Our main theorem (see theorem 8.1) can be formulated as follows:

*Supported in part by NSF grant DMS-0505778. 
Theorem 1.2. Let $Z$ be a symplectic surface in $\bar{X}$ homologous to a section. Consider the homology class of sections disjoint from $Z$ and suppose that it has positive symplectic area. Then the set of symplectic surfaces in $\bar{X}$ belonging to this homology class and avoiding $Z$ is non-empty and (weakly) contractible.

In particular the statement on local isotopy is an easy consequence of this. We will discuss ruled symplectic 4-manifolds in more detail in section 3, we also postpone till this section the section-by-section description of the rest of the paper. Similar results have already been obtained by Joseph Coffey (see [3]), and in fact the current version of this paper is highly motivated by his results.

The group of symplectomorphisms of $\bar{X}$ acts on the set of symplectic surfaces in $\bar{X}$ and this leads to an interesting connection between the two. In particular we can obtain some results in the spirit of [1]. This is briefly discussed in section 9 .

Unless stated otherwise, we will always mean that a surface is embedded, oriented and non-parameterized, any family is smoothly dependent on parameters, and homotopical properties such as homotopy equivalence or contractibility are to be understood in the weak sense (that is, weak homotopy equivalence or weak contractibility).

Acknowledgments. The second author would like to thank Shengda $\mathrm{Hu}$, Octavian Cornea and François Lalonde for many helpful discussions on the subject, Joseph Coffey for explaining his relating work and referring us to his paper, and Paul Biran and Leonid Polterovich for a chance to present the previous approach to the problem on Geometry and Dynamics seminar and helpful comments received therein.

\section{Standard symplectic disk and sphere bundles}

Standard symplectic disk bundles:

Given a Riemann surface $\Sigma$ of genus $g$ and $d \in \mathbb{Z}$, we will construct the most canonical symplectic disk bundle $(X, \omega)$ of degree $d$ over $\Sigma$ (see also [2]).

Denote by $L$ the complex line bundle over $\Sigma$ with Chern number $d$, thus $[\Sigma] \in H_{2}(L)$ satisfies $[\Sigma] \cdot[\Sigma]=d$. Let $r$ denote the radial coordinate on $L$ with respect to a Hermitian metric on $L$, let $\pi: L \rightarrow \Sigma$ denote the natural projection to the base, let $\Sigma_{0}$ denote the 0 -section, and let $P=\{r=1\} \subset L$ denote the unit circle bundle.

First suppose that $d \neq 0$. Fix an area form $\sigma$ on $\Sigma$ with $\int_{\Sigma} \sigma=|d|$. We can choose an $S^{1}$-invariant connection 1 -form $\alpha$ on $P$ so that $d \alpha=\pi^{*} \sigma$, and extend $\alpha$ to the complement of the 0 -section of $L$ as $\pi^{*} \alpha$. In the case $d>0$, we let $X=\{r<1\} \subset L$ be the unit disk bundle and define $\omega=\pi^{*} \sigma-d\left(r^{2} \alpha\right)$. In the case $d<0$, we let $X=L$ and define $\omega=\pi^{*} \sigma+d\left(r^{2} \alpha\right)$. Note that in both cases on the complement of the 0 -section the symplectic form can be written as $\omega=d\left(\left(1 \pm r^{2}\right) \alpha\right)$.

In the case $d=0$, we let $X=\Sigma \times \mathbb{C}$ be the trivial line bundle over $\Sigma$. We fix an area form $\sigma$ on $\Sigma$ of total area 1, and let $\tau$ be the standard 
symplectic form on $\mathbb{R}^{2}=\mathbb{C}$. Define $\omega=\sigma \oplus \tau$.

In all cases, $\omega$ is a symplectic form on $X$, and the fibers of $X$ and the 0 -section of $X$ are $\omega$-symplectic.

Symplectic neighborhood theorem:

Let $(M, \omega)$ be any 4-dimensional symplectic manifold and let $\Sigma \subset$ $M$ be a sympectic surface. By a symplectic neighborhood theorem, a neighborhood of $\Sigma$ is determined by the area $\int_{\Sigma} \omega$ and the self-intersection number $d=\Sigma \cdot \Sigma$, the latter being also equal to the first Chern number of the symplectic normal bundle to $\Sigma$.

Thus in a neighborhood $U$ of $\Sigma$ an appropriate multiple $c \omega$ of the symplectic form $\omega$ can be written in a standard form described above:

$$
c \omega= \begin{cases}\pi^{*} \sigma-d\left(r^{2} \alpha\right)=d\left(\left(1-r^{2}\right) \alpha\right) & \text { if } d>0 \\ \pi^{*} \sigma+d\left(r^{2} \alpha\right)=d\left(\left(1+r^{2}\right) \alpha\right) & \text { if } d<0 \\ \pi^{*} \sigma+d y_{1} \wedge d y_{2} & \text { if } d=0\end{cases}
$$

where $\sigma$ is an area form on $\Sigma$ normalized so that the total area of $\Sigma$ equals $|d|$ if $d \neq 0$ and equals 1 if $d=0, \pi: U \rightarrow \Sigma$ is a projection map, and $\alpha$ (if $d \neq 0$ ) is a connection 1-form with $d \alpha=\pi^{*} \sigma$.

Moreover the same theorem with parameters applies to any compact family of $\omega_{\lambda}$-symplectic surfaces $\Sigma_{\lambda}$ to produce a family of such standard presentations of $\omega_{\lambda}$ in sufficiently small neighborhoods of $\Sigma_{\lambda}$.

Compactifying standard disk bundles to standard sphere bundles:

Given a standard symplectic disk bundle $X$ over $\Sigma$, let $X(R)=\{r \leq$ $R$, where $R<1$ in the case $d>0$.

If $d \neq 0$ then by symplectic cutting (see for example [2] for a proof) one can compactify the symplectic disk bundle $(X(R), \omega)$ to a symplectic sphere bundle without changing the area of the fiber. Slightly abusing notation, we denote this sphere bundle by $(\bar{X}(R), \omega)$. Roughly speaking this construction collapses the boundary of $X(R)$ to a symplectic section $\Sigma_{\infty}$ of $\bar{X}$, in particular $\left(\bar{X}(R) \backslash \Sigma_{\infty}, \omega\right)$ is symplectomorphic to $(X(R), \omega)$.

If $d=0$, we note that a standard symplectic disk $(D(R), \tau)$ can be compactified to a symplectic sphere, and so trivial symplectic disk bundle $(X(R), \omega=\sigma \oplus \tau)$ can be compactified to a trivial symplectic sphere bundle $(\bar{X}(R), \omega=\sigma \oplus \tau)$. When clear, we omit the parameter $R$ from the notation.

The sphere bundles that we obtain are examples of ruled symplectic 4manifolds discussed in the next section. Note that up to a diffeomorphism there are precisely two sphere bundles over a surface of genus $g$ : a trivial one and a nontrivial one.

\section{Ruled symplectic 4-manifolds}

We recall that a symplectic 4-manifold $(\bar{X}, \omega)$ is called ruled if it is a fibration over a Riemann surface with fiber $S^{2}$. By choosing the right orientations of the base and of the fiber of the fibration, we will always assume that the integral of $\omega$ over the fiber and the integral of $\omega^{2}$ over $\bar{X}$ are positive. Up to a symplectomorphism ruled 4-manifolds have been 
classified by F. Lalonde and D. McDuff 8, 9] who showed that any two cohomologous symplectic forms on $\bar{X}$ are in fact diffeomorphic.

What makes understanding symplectic properties of ruled 4-manifolds easier is that for a large class $\mathcal{J}$ of $\omega$-tame almost complex structures (all $\omega$-tame almost complex structures if $g>0$ ) $\bar{X}$ admits a holomorphic foliation by smooth embedded spheres representing the homology class of the fiber. For a general theory of (pseudo)holomorphic curves we refer the reader to 5$] 12$.

We introduce several notations which will be used throughout the paper. We denote by $F$ the homology class of a fiber and by $A_{d}$ the homology class of a section with self-intersection $d \in \mathbb{Z}$. We note that if up to a diffeomorphism $\bar{X}$ is a trivial bundle over $\Sigma$ then all homology classes $A_{d}$ with $d$ even can be represented by embedded surfaces in $\bar{X}$, and if $\bar{X}$ is a nontrivial bundle - then all homology classes $A_{d}$ with $d$ odd. We also note that $H_{2}(\bar{X} ; \mathbb{R})$ is generated by $F$ and $A_{d}$ for any $d \in \mathbb{Z}$, and $F \cdot F=0$, $A_{d} \cdot A_{d}=d, A_{d} \cdot F=1$. We let $a_{d}(\omega)$ and $f(\omega)$ denote the integrals of $\omega$ on the homology classes $A_{d}$ and $F$ respectively.

For a fixed $d \in \mathbb{Z}$ we will say that $(\bar{X}, \omega)$ satisfies the cohomology assumption $\left(*_{d}\right)$ if:

- either $g \neq 0$ or $d \neq 0$,

- $a_{d}(\omega)>0$ and $a_{-d}(\omega)>0$.

When the symplectic form is understood from the context, we will just write $a_{d}$ and $f$.

The standard symplectic sphere bundles of degree $d$ we obtained in the previous section by compactification automatically satisfy this assumption if either $d \neq 0$ or $g \neq 0$ since both sections $\Sigma_{0}$ and $\Sigma_{\infty}$ are $\omega$-symplectic. For a more general ruled 4-manifold this assumption just means that both homology classes $A_{d}$ and $A_{-d}$ can be represented by sympectic surfaces.

As mentioned in the introduction, the main goal of this paper is to understand the set of symplectic surfaces in $\bar{X}$. We denote by $\mathcal{S}_{d}(\bar{X}, \omega)$ the set of $\omega$-symplectic surfaces in $\bar{X}$ representing the homology class $A_{d}$, and we denote by $\mathcal{S}(\bar{X}, \omega)$ the union of $\mathcal{S}_{d}(\bar{X}, \omega)$ over all $d \in \mathbb{Z}$. As follows from the classification of ruled 4-manifolds, a necessary and sufficient condition for $\mathcal{S}_{d}(\bar{X}, \omega)$ to be nonempty is that $a_{d}(\omega)>0$. When clear from the context, we will omit the arguments $\bar{X}$ or $\omega$ from the notation. Given a symplectic surface $Z \in \mathcal{S}_{-d}(\bar{X}, \omega)$, we will also denote by $\mathcal{S}_{d}(\bar{X} \backslash Z, \omega)$ the set of symplectic surfaces in $\mathcal{S}_{d}(\bar{X}, \omega)$ disjoint from $Z$.

We describe the contents of later sections of this paper. In section 4 we prove a theorem on the existence of holomorphic foliations of $\bar{X}$ by spheres in the homology class $F$ - this is very well-known if $g \geq 1$, but possibly our version is slightly uncommon in the case $g=0$. A direct consequence of this existence is the proof in section 5 that any $S^{n}$-family $\Sigma_{\lambda} \subset \mathcal{S}(\bar{X} \backslash Z, \omega)$ of symplectic surfaces disjoint from a symplectic surface $Z \in \mathcal{S}_{-d}(\bar{X}, \omega)$ can be contracted through smoothly embedded surfaces.

Given a symplectic surface $\Sigma \in \mathcal{S}_{d}$, a standard presentation (11) of the symplectic form in a neighborhood of $\Sigma$, and a symplectic foliation $\mathcal{F}$ of $\bar{X}$ with all leaves intersecting $\Sigma$ positively and transversely, we show in section 6 how to modify this foliation near $\Sigma$ so that its leaves near $\Sigma$ 
coincide with the fibers of $\pi$. We will say that $\mathcal{F}$ nicely intersects $\Sigma$ to denote this phenomenon.

Many properties of ruled symplectic 4-manifolds have been obtained using inflation. Inflation is a procedure which works in any symplectic 4-manifold $(M, \omega)$ and which consists of altering the cohomology class of $\omega$ by adding to it multiples of the Thom class of a symplectic surface in $M$ of nonnegative self-intersection (see Lalonde-McDuff [8] and McDuff [10 11).

Inflation first appeared in the papers on the classification of symplectic structures on ruled 4-manifolds (see 8, 9]) where the authors use it in a combination with another idea. We give a very rough sketch of this. Let $(\bar{X}, \omega)$ be a ruled symplectic 4-manifold, let $J \in \mathcal{J}$ be an almost complex structure, let $\mathcal{F}$ be the corresponding $J$-holomorphic foliation of $\bar{X}$ by spheres in the homology class $F$, and let $\Sigma$ be a symplectic section of $\mathcal{F}$. Consider other symplectic forms on $\bar{X}$ which have the form $\omega+k \pi^{*} \sigma$, where $\pi: \bar{X} \rightarrow \Sigma$ denotes the projection along $\mathcal{F}, \sigma$ is an area form on $\Sigma$, and $k \geq 0$ is any nonnegative constant. It is easy to check that the forms $\omega_{k}=\omega+k \pi^{*} \sigma$ are indeed symplectic for any $k \geq 0$ : $\omega_{k} \wedge \omega_{k}=\omega^{2}+2 k \omega \wedge \pi^{*} \sigma$ and both terms evaluate positively on a positively oriented basis of tangent vectors. However the cohomology classes of $\omega_{k}$ are now different from the cohomology class of $\omega$. Under certain conditions one can inflate along a suitable symplectic surface, obtaining symplectic forms $\omega_{k}^{\prime}$ whose cohomology classes are multiples of $[\omega]$. Rescaling the symplectic forms, Moser's argument can be applied to produce interesting results.

In section 7 we prove a version of the above argument which has the advantages that no assumption on the self-intersection of surfaces is made and that it applies nicely to families. This will allow to prove the main theorem stated in the introduction. This proof and its simple corollaries are discussed in section 8 .

\section{Holomorphic foliations}

Let $\mathcal{J}$ be the set of $\omega$-tame almost complex structures on $\bar{X}$. For a symplectic surface $\Sigma \in \mathcal{S}_{d}(\bar{X})$ we denote by $\mathcal{J}_{\Sigma} \subset \mathcal{J}$ the subset of almost complex structures for which $\Sigma$ is holomorphic. For $J \in \mathcal{J}$ and a homology class $A \in H_{2}(M)$ we define $\mathcal{M}(J ; A)$ as the moduli space of irreducible $J$-holomorphic spheres representing the homology class $A$. We abbreviate $\mathcal{M}(J ; F)$ as $\mathcal{M}(J)$.

By [7, for any $J \in \mathcal{J}, \mathcal{M}(J)$ is a smooth manifold. If nonempty, then by the index formula its dimension is 2 . Since $F \cdot F=0$, different spheres in $\mathcal{M}(J)$ cannot intersect by the positivity of intersection.

Proposition 4.1. Let $(\bar{X}, \omega)$ be a ruled symplectic 4 -manifold. Let $J \in$ $\mathcal{J}_{\Sigma}$

- Suppose that either $g \geq 1$, or that $g=0, d \neq 0$ and the cohomology assumption $\left(*_{d}\right)$ is satisfied. Then the spheres in $\mathcal{M}(J)$ form a holomorphic foliation of $\bar{X}$. Moreover, each such sphere intersects the surface $\Sigma$ uniquely and transversely. Furthermore, these foliations depend smoothly on $J$. 
- If $g=d=0$, then the spheres in $\mathcal{M}\left(J ; A_{0}\right)$ form a holomorphic foliation of $\bar{X}=S^{2} \times S^{2}$. Moreover, each such foliation contains the sphere $\Sigma$ and these foliations depend smoothly on $J$.

Proof. Consider the first part of the proposition. Given that the spheres in $\mathcal{M}(J)$ form a foliation of $\bar{X}$, the second statement follows from the positivity of intersection with $\Sigma$ and the third from the general theory of holomorphic curves (see [5] 12]). Now, the fact that for $J \in \mathcal{J}$ the $J$-holomorphic spheres in $\mathcal{M}(J)$ produce a foliation of $\bar{X}$ is well-known when $g>0$, or when $g=0, d \neq 0$ and $J \in \mathcal{J}$ is generic (that is away from a subset of codimension 2), see 12. Thus it remains to prove the first statement only in the case $g=0, d \neq 0$ under the cohomology assumption $a_{d}>0$ and $a_{-d}>0$.

Assuming $g=0, d \neq 0$, let $J_{n} \in \mathcal{J}$ be a sequence of generic almost complex structures approximating $J \in \mathcal{J}_{\Sigma}$. By compactness theorem there is a (possibly) cusp $J$-holomorphic curve through every point of $\bar{X}$ which represents the homology class $F$. We prove by contradiction that such a curve must in fact be nondegenerate. We break the proof into two cases.

Case $d>0$.

Suppose that $C=\cup_{i=1}^{n} C_{i}$ is a cusp $J$-holomorphic curve representing the homology class $F$ and $n \geq 2$. Write $\left[C_{i}\right]=k_{i} A_{-d}+m_{i} F$. Thus $F=$ $\sum_{i=1}^{n}\left(k_{i} A_{-d}+m_{i} F\right)$, with $\sum k_{i}=0$ and $\sum m_{i}=1$. By the cohomology assumption, $\omega([\Sigma])=a_{d}=a_{-d}+d \cdot f>f$ and so no component $C_{i}$ can be a multiple cover of $\Sigma$. Thus by the positivity of intersection with $\Sigma, m_{i} \geq 0$ for all $i$. It follows that exactly one of the $m_{i}$ 's is 1 (we denote this $m_{i}$ by $m_{1}$ ) and the rest are zero. Also note that $k_{1}<0$, for otherwise $\omega\left(\left[C_{i}\right]\right)>f$. Thus the splitting in homology becomes $F=$ $\left(F-k A_{-d}\right)+\sum_{i=2}^{n} k_{i} A_{-d}$, where $k=-k_{1}=\sum_{i=2}^{n} k_{i}>0$ and $k_{i} \geq 0$ for $i \geq 2$.

We note that $F \cdot\left(F-k A_{-d}\right)=-k<0$ and so there cannot be any non-cusp $J$-holomorphic spheres representing the class $F$. Choose a point $p$ not on $C$. There must exist a cusp $J$-holomorphic sphere $C^{\prime}$ through $p$ representing $F$. Repeating previous arguments, $C^{\prime}$ must be also split as $\cup_{i=1}^{n^{\prime}} C_{i}^{\prime}$, with $\left[C_{1}^{\prime}\right]=F-k^{\prime} A_{-d}, k^{\prime}>0$. However, $\left(F-k A_{-d}\right) \cdot(F-$ $\left.k^{\prime} A_{-d}\right)=-k-k^{\prime}-k k^{\prime} d<0$, which is a contradiction since both $C_{1}$ and $C_{1}^{\prime}$ are $J$-holomorphic.

Case $d<0$.

Again, suppose that $C=\cup C_{i}$ is a cusp $J$-holomorphic curve in the homology class $F$. Since $\Sigma^{2}=d<0$, any holomorphic curve whose homology class is a multiple of $A_{d}$ must be geometrically a multiple cover of $\Sigma$. We group together those components which represent multiple covers of $\Sigma$ and those which do not. Thus in homology $F=\sum_{j=1}^{n} s_{j} A_{d}+\sum_{i=1}^{\tilde{n}}\left(k_{i} A_{-d}+\right.$ $m_{i} F$ ) with $\tilde{n}>0$ and no component in the second sum is a multiple cover of $\Sigma$. By the positivity of intersections with $\Sigma, m_{i} \geq 0$ for all $i$. By the cohomology assumption $a_{-d}=a_{d}+|d| \cdot f$, in particular $a_{-d}>f$. Hence for every component in the second sum, $m_{i} \geq 1$ and $k_{i}<0$. Clearly $s_{j}>0$ for all $j$. Let $\bar{k}_{i}=-k_{i}>0$. Since $A_{d}=A_{-d}+d F, \sum s_{j}=\sum \bar{k}_{i}$ and $1+\sum s_{j} d=\sum m_{i}$. 
Since the area of each component must be positive, $\omega\left(m_{i} F-\bar{k}_{i} A_{-d}\right)=$ $m_{i} f-\bar{k}_{i} a_{-d}>0$. Recall that $a_{-d} / f>|d|$ and so $m_{i} \geq \bar{k}_{i}|d|+1$. Adding this inequalities for $i=1, \ldots \tilde{n}$, we get

$$
\sum m_{i} \geq \tilde{n}+\sum \bar{k}_{i}|d|=\tilde{n}+\sum s_{j}|d|=\tilde{n}-1+\sum m_{i} .
$$

It follows that $\tilde{n}-1 \leq 0$ and so the second sum consists of an exactly one term. Let $k=\bar{k}_{1}, m=m_{1}$, then $\sum s_{j}=k>0$ and $m_{1}=1-k d$. Thus in homology the splitting of $C$ must be of the form $F=(k \Sigma)+((1-k d) F-$ $\left.k A_{-d}\right)$ with $(1-k d) F-k A_{-d}$ represented by a $J$-holomorphic sphere.

Note that $\left((1-k d) F-k A_{-d}\right) \cdot F=-k<0$, and so there cannot be any non-cusp $J$-holomorphic spheres in the class $F$. Choose a point $p$ not on $C$ and not on $\Sigma$, and let $C^{\prime}$ be a cusp $J$-holomorphic curve through $p$ representing $F$. By the above arguments $C^{\prime}$ must contain a component $C_{1}^{\prime}$ with homology $\left(1-k^{\prime} d\right) F-k^{\prime} A_{-d}$ where $k^{\prime}>0$. We note that $\left.C_{1} \cdot C_{1}^{\prime}=\left((1-k d) F-k A_{-d}\right) \cdot\left(\left(1-k^{\prime} d\right) F-k^{\prime} A_{-d}\right)\right)=-k\left(1-k^{\prime} d\right)-$ $k^{\prime}(1-k d)-k k^{\prime} d=-k-k^{\prime}+k k^{\prime} d<0$, contradicting to the positivity of intersection.

Consider the second part of the proposition. We note that since $\Sigma$ is $J$-holomorphic, there cannot be any cusp $J$-holomorphic spheres representing the homology class $A_{0}$. The existence of the required foliation and its smooth dependence on $J$ now follow by standard methods.

\section{Diffeomorphisms and smooth isotopy}

Assume that $(\bar{X}, \omega)$ is a ruled symplectic 4-manifold, that $\Sigma \subset \mathcal{S}_{d}(\bar{X})$ is a symplectic surface, and that the conclusions of the first part of proposition 4.1 hold. In other words, assume that for every $J \in \mathcal{J}_{\Sigma}$ the spheres in $\mathcal{M}(J)$ form a holomorphic foliation of $\bar{X}$. This is automatically satisfied if one imposes the cohomology assumption $\left(*_{d}\right)$, but this is also satisfied when $g=d=0$ and $f(\omega) \leq a_{0}(\omega)$.

We will write $\mathcal{F}(J)$ for the foliation given by $\mathcal{M}(J)$. Note that we have a canonical diffeomorphism $\mathcal{M}(J) \rightarrow \Sigma$ associating to a holomorphic sphere in $\mathcal{M}(J)$ its point of intersection with $\Sigma$.

Suppose that $J_{t}, t \in[0,1]$, is a family of almost complex structures in $\mathcal{J}_{\Sigma}$. Let $\mathcal{F}_{t}=\mathcal{F}\left(J_{t}\right)$ be the family of corresponding foliations. For $p \in \Sigma$ we denote by $C_{t, p}$ the sphere in $\mathcal{F}_{t}$ intersecting $\Sigma$ at $p$. We will define a canonical vector field $Y_{t}$ generating a family $\Phi_{t}$ of diffeomorphisms of $\bar{X}$ mapping the spheres $C_{0, p}$ to the spheres $C_{t, p}$. Denote by $N_{t, p}$ the symplectic normal bundle to $C_{t, p}$. For each $t$ and $p$, for $|\epsilon|$ sufficiently small, $J_{t+\epsilon}$-holomorphic spheres $C_{t, p}$ can be viewed as sections $\Gamma_{t, p, \epsilon}$ of $N_{t, p}$. Define $Y_{t}(p)=\left.\frac{d}{d \epsilon}\right|_{\epsilon=0} \Gamma_{t, p, \epsilon}$ (note that $Y_{t}$ vanishes on $\Sigma$ ) and let $\Phi_{t}$ be the time $t$ flow of $Y_{t}, 0 \leq t \leq 1$. Thus $\Phi_{t}$ maps the spheres $C_{0, p} \in \mathcal{F}_{0}$ to the corresponding spheres $C_{t, p} \in \mathcal{F}_{t}$, and $\Phi_{t}=i d$ on $\Sigma$.

We are now ready to prove the smooth version of the main theorem (see theorem [1.2):

Lemma 5.1. Let $(\bar{X}, \omega)$ be a ruled symplectic 4-manifold. Fix d and suppose that the cohomology assumption $\left(*_{d}\right)$ holds. Let $\Sigma \subset \mathcal{S}_{d}(\bar{X}, \omega)$ be a symplectic surface. Let $\Sigma_{\lambda} \subset \mathcal{S}_{-d}(\bar{X} \backslash \Sigma, \omega)$ be a family of symplectic 
surfaces parameterized by $\lambda \in S^{n}, n \geq 0$. Then the family $\Sigma_{\lambda}$ can be contracted through smooth surfaces within $\bar{X} \backslash \Sigma$.

Proof. Given a family of symplectic surfaces $\Sigma_{\lambda}, \lambda \in S^{n}$, we can choose a family $J_{\lambda} \in \mathcal{J}_{\Sigma}$ of almost complex structures such that for each $\lambda$ the surface $\Sigma_{\lambda}$ is $J_{\lambda}$-holomorphic.

Since $\mathcal{J}_{\Sigma}$ is contractible, we can contract the loop $J_{\lambda}$ to any given almost complex structure $J_{1} \in \mathcal{J}_{\Sigma}$. In other words, there exists a family $J_{\lambda, t} \subset \mathcal{J}_{\Sigma}, t \in[0,1]$, so that

1. $J_{\lambda, 0}=J_{\lambda}$

2. $J_{\lambda, 1}=J_{1}$.

Since the assumptions of proposition 4.1 are satisfied, for each $\lambda$ and $t$ there exists a foliation $\mathcal{F}_{\lambda, t}=\mathcal{F}\left(J_{\lambda, t}\right)$ of $\bar{X}$ by holomorphic spheres in the homology class $F$. Moreover, $\mathcal{F}_{\lambda, t}$ depends smoothly on the two parameters. We let $\mathcal{F}_{1}=\mathcal{F}\left(J_{1}\right)$ be the foliation corresponding to $J_{1}$. Fixing $\lambda$, we define the canonical vector field $Y_{\lambda, t}$ whose time $t$ flow $\Phi_{\lambda, t}$ maps the foliation $\mathcal{F}_{\lambda, 0}$ to the foliation $\mathcal{F}_{\lambda, t}$ and which is $i d$ on $\Sigma$. Note that by positivity of intersections, each surface $\Sigma_{\lambda}$ intersects every sphere in $\mathcal{F}_{\lambda, 0}$ uniquely and transversely, and thus can be viewed as a section of the foliation $\mathcal{F}_{\lambda, 0}$. Define $\Sigma_{\lambda, t}=\Phi_{\lambda, t}\left(\Sigma_{\lambda}\right)$. Then $\Sigma_{\lambda, t}$ a section of $\mathcal{F}_{\lambda, t}$ for every $\lambda$ and $t$, and $\Sigma_{\lambda, t}$ provides a smooth isotopy between the $S^{n}$-family $\Sigma_{\lambda}=\Sigma_{\lambda, 0}$ and an $S^{n}$-family of sections $\Sigma_{\lambda, 1}$ of $\mathcal{F}_{1}$. By construction, all the surfaces $\Sigma_{\lambda, t}$ are disjoint from $\Sigma$. Since the set of sections of $\mathcal{F}_{1}$ which are disjoint from $\Sigma$ is contractible, the $S^{n}$-family $\Sigma_{\lambda, 1}$ can be contracted to any given section of $\mathcal{F}_{1}$ disjoint from $\Sigma$ through similar sections. This provides the required smooth contraction of $\Sigma_{\lambda}$.

For the purposes of section 8 we will still denote the extended isotopy from $\Sigma_{\lambda}$ to a section of $\mathcal{F}_{1}$ by $\Sigma_{\lambda, t}$. We can extend the initial family $\mathcal{F}_{\lambda, t}$ of foliations by a constant family $\mathcal{F}_{1}$. Since all the objects corresponding to $(\lambda, 1)$ are identified, we can think of a pair $\mu=(\lambda, t)$ as of a point in $B^{n+1}$.

\section{Straightening foliations}

We show how to modify symplectic foliations so that they intersect with symplectic surfaces nicely.

Lemma 6.1. Let $(\bar{X}, \omega)$ be a ruled symplectic 4-manifold. Let $\Sigma \subset \bar{X}$ be a symplectic surface and fix a standard presentation (1) of $\omega$ near $\Sigma$. Given any symplectic foliation of $\bar{X}$ intersecting $\Sigma$ positively and transversely, one can modify it in a (arbitrarily small) neighborhood of $\Sigma$ so that the new foliation coincides with the foliation by fibers of $\pi$ in some (even smaller) neighborhood of $\Sigma$.

Proof. Fix a point $p \in \Sigma$. Denote by $C_{p}$ the curve in the foliation intersecting $\Sigma$ at $p$ and denote by by $F_{p}$ the fiber of $\pi$ through $p$ (which is defined in a neighborhood of $\Sigma$ ).

We first consider the case $d=\Sigma \cdot \Sigma=0$. In a neighborhood $U \times$ $V$ of $p$ the symplectic form can be written as $\omega=d x_{1} \wedge d x_{2}+d y_{1} \wedge$ 
$d y_{2}=d x_{1} \wedge d x_{2}+r d r \wedge d \theta$, where $\left(x_{1}, x_{2}\right) \in U \subset \Sigma$ are coordinates on a neighborhood $U$ of $p$ on $\Sigma,\left(y_{1}, y_{2}\right) \in V \subset F_{p}$ are rectilinear coordinates on a neighborhood $V$ of $p$ on $F_{p}$, and $(r, \theta)$ are the corresponding polar coordinates on $V$.

By restricting to a possibly smaller neighborhood $U \times V$ we can assume that $C_{p} \cap(U \times V)$ is a graph over $V$, thus in $\left(r, \theta, x_{1}, x_{2}\right)$-coordinates $C_{p}$ has the form $C_{p}=\left\{\left(r, \theta, x_{1}(r, \theta), x_{2}(r, \theta)\right) \mid(r, \theta) \in V\right\}$. Let $q \in C_{p} \cap(U \times V)$. Notice that since $C_{p}$ intersects $\Sigma$ positively and transversely, the restriction of the form $r d r \wedge d \theta$ to $T_{q} C_{p}$ induces positive orientation. The tangent space $T_{q} C_{p}$ (when $q \neq p$ ) is spanned by $e_{1}=\left(1,0, \frac{\partial x_{1}}{\partial r}(r, \theta), \frac{\partial x_{2}}{\partial r}(r, \theta)\right)$ and $e_{2}=\left(0,1, \frac{\partial x_{1}}{\partial \theta}(r, \theta), \frac{\partial x_{2}}{\partial \theta}(r, \theta)\right)$. Since $T_{q} C_{p}$ is symplectic, for $r>0$ we have that

$$
\omega\left(e_{1}, e_{2}\right)=r+D(r, \theta)>0,
$$

where $D(r, \theta)=\left[\frac{\partial x_{1}}{\partial r} \frac{\partial x_{2}}{\partial \theta}-\frac{\partial x_{2}}{\partial r} \frac{\partial x_{1}}{\partial \theta}\right](r, \theta)$. Since $C_{p}$ is smooth and symplectic at $p$, we have that $\lim _{(r, \theta) \rightarrow 0}(1+D(r, \theta) / r)$ exists and is positive. Since $V$ is compact, there is a constant $\delta>0$ so that

$$
(1-\delta) r+D(r, \theta)>0
$$

for $r>0$.

Given $\epsilon_{1}>0$ sufficiently small, and given $\epsilon_{0}>0$ sufficiently small compared to $\epsilon_{1}$, one can choose a nonnegative nondecreasing function $\beta(r)$ so that $\beta(r)=0$ for $r \leq \epsilon_{0}, \beta(r)=r$ for $r \geq \epsilon_{1}, \beta(r) \leq r$ and $(1-\delta) \beta^{\prime}(r)<1$.

We choose $\epsilon_{1}>0$ sufficiently small so that $\left\{r \leq \epsilon_{1}\right\} \subset V$ and so that it satisfies whatever smallness assumptions of the lemma. We choose $\epsilon_{0}$ and $\beta(r)$ as in the preceding paragraph. We define the curve $\tilde{C}_{p}$ by "radially-rescaling" $C_{p}: \tilde{C}_{p}=\left\{\left(r, \theta, x_{1}(\beta(r), \theta), x_{2}(\beta(r), \theta)\right) \mid(r, \theta) \in V\right\}$. We note that $\tilde{C}_{p}$ defined in this way is a smooth sphere which coincides with $F_{p}$ for $r \leq \epsilon_{0}$ and which coincides with $C_{p}$ for $r \geq \epsilon_{1}$. We claim that $\tilde{C}_{p}$ is $\omega$-symplectic. We only need to verify this for $\epsilon_{0}<$ $r<\epsilon_{1}$. For $q \in \tilde{C}_{p}$ lying above $(r, \theta) \in F_{p}$, the tangent plane $T_{q} \tilde{C}_{p}$ is spanned by $\tilde{e}_{1}=\left(1,0, \beta^{\prime}(r) \frac{\partial x_{1}}{\partial r}(\beta(r), \theta), \beta^{\prime}(r) \frac{\partial x_{2}}{\partial r}(\beta(r), \theta)\right)$ and $\tilde{e}_{2}=$ $\left(0,1, \frac{\partial x_{1}}{\partial \theta}(\beta(r), \theta), \frac{\partial x_{2}}{\partial \theta}(\beta(r), \theta)\right)$. Thus at $q$ we have:

$$
\omega_{q}\left(\tilde{e}_{1}, \tilde{e}_{2}\right)=r+D(\beta(r), \theta) \beta^{\prime}(r) \geq r-(1-\delta) r \beta^{\prime}(r)>0,
$$

verifying that $\tilde{C}_{p}$ is symplectic (at $q$ ).

Next, for each $p^{\prime} \in \Sigma$ we let $C_{p^{\prime}}$ be the curve in the foliation through $p^{\prime}$ and we let $F_{p^{\prime}}$ be the fiber through $p^{\prime}$. We note that for $p^{\prime}$ sufficiently close to $p, C_{p^{\prime}}$ is a graph over $F_{p^{\prime}} \cap(U \times V)$. Moreover (being symplectic is an open condition), for $p^{\prime}$ sufficiently close to $p$ the radially-rescaled curves $\tilde{C}_{p^{\prime}}$ (defined in the analogous way with the same function $\beta(r)$ ) are also $\omega$-symplectic. By a compactness argument one can choose the same $\beta$ to work for every sphere in the foliation. Thus the "radially-rescaled" spheres $\tilde{C}_{p}$ depend smoothly on $p$ and satisfy all the requirements of the lemma.

The proof is almost analogous in the case $d \neq 0$. Since $T_{p} \Sigma$ and $T_{p} F_{p}$ are $\omega$-orthogonal, $\omega$ has the form $\omega_{p}=d x_{1} \wedge d x_{2}+r d r \wedge d \theta$ at the 
point $p$ and is sufficiently close to this form in a sufficiently small neighborhood $U \times V$ of $p$. More precisely, for $q \in C_{p}$ in a sufficiently small neighborhood $U \times V$ of $p$ the planes $T_{q} C_{p}$ are $\omega_{p}$-symplectic. By the previous construction we can define an $\omega_{p}$-symplectic "radially-rescaled" curve $\tilde{C}_{p}$. The tangent planes to $\tilde{C}_{p}$ are $\omega_{q}$-symplectic for all $q$ sufficiently close to $p$. Thus shrinking the defining function $\beta(r)$ if necessary (i.e. considering $\frac{1}{K} \beta(K r)$ for $K$ sufficiently large), we can obtain the required $\omega$-symplectic rescaling of $C_{p}$. The rest of the proof follows by a compactness argument.

Remark 6.2. Suppose that $\Sigma_{\lambda}, \lambda \in S^{n}$, is a family of $\omega$-symplectic surfaces in $\bar{X}$, and $\mathcal{F}_{\lambda}$ is a family of $\omega$-symplectic foliations of $\bar{X}$ such that for each $\lambda$ every sphere in $\mathcal{F}_{\lambda}$ intersects $\Sigma_{\lambda}$ positively and transversely. We fix a family of standard presentations of $\omega$ near $\Sigma_{\lambda}$ and denote by $\pi_{\lambda}$ the corresponding family of projection maps (each defined in a neighborhood of the corresponding surface). Clearly one can choose the same function $\beta(r)$ for every sphere in every foliation. Thus we can obtain a new family $\mathcal{F}_{\lambda}$ of $\omega$-symplectic foliations so that for each $\lambda$ the foliation $\mathcal{F}_{\lambda}$ intersects $\Sigma_{\lambda}$ nicely.

\section{An inflation-like argument}

The heart of this paper lies in the following simple argument.

Proposition 7.1. Let $(\bar{X}, \omega)$ be a ruled symplectic 4-manifold. Let $\Sigma \subset$ $\mathcal{S}_{d}(\bar{X})$ be a symplectic surface and fix a standard presentation (1) of $\omega$ near $\Sigma$. By scaling $\omega$ we may assume that $a_{d}(\omega)=|d|$ if $d \neq 0$ and $a_{d}(\omega)=1$ if $d=0$. If $d>0$ also assume that the cohomology assumption $\left(*_{d}\right)$ is satisfied. Let $\mathcal{F}$ be a symplectic foliation of $\bar{X}$ nicely intersecting $\Sigma$. Then there exists a family $\hat{\omega}_{k}, k \geq 0$, of cohomologous symplectic forms on $\bar{X}$ which smoothly depend on the parameter $k$ and which satisfy:

- $\hat{\omega}_{k}=\omega$ near $\Sigma$,

- $\hat{\omega}_{k}$ is a multiple of $\omega+k \pi^{*} \sigma$ outside $U$,

- $\hat{\omega}_{k}=\omega$ when $k=0$.

Proof. Recall that for $k \geq 0$ the forms $\omega_{k} \stackrel{\text { def }}{=} \omega+k \pi^{*} \sigma$ are symplectic. We break the construction into 3 cases.

Case: $d>0$ :

Define the form $\omega_{k}^{\prime}$ by rescaling the form $\omega_{k}$ :

$$
\omega_{k}^{\prime}=\lambda_{k} \omega_{k}
$$

where

$$
\lambda_{k}=\frac{1-f}{1+k-f} .
$$

Note that $\lambda_{k} \in(0,1]$ and $a_{-d}\left(\omega_{k}^{\prime}\right)=a_{-d}(\omega)$.

Choose $\epsilon$ so that $\{r \leq \epsilon\} \subset U$. Let $\beta(r)$ be a positive decreasing function which equals $1-r^{2}$ near $r=0$ and equals $\frac{1-f}{k+1-f}\left(k+1-r^{2}\right)$ near $\epsilon$. To verify that such a function exists, notice that when $k=0$ 
the expressions $\frac{1-f}{k+1-f}\left(k+1-r^{2}\right)$ and $1-r^{2}$ coincide, and when $k>0$ the expression $1-r^{2}$ is strictly greater than $\frac{1-f}{k+1-f}\left(k+1-r^{2}\right)$, at least for all $r$ sufficiently small. The function $\beta(r)$ can be constructed by an appropriate smoothing between these two functions.

Define the form $\hat{\omega}_{k}=d(\beta(r) \alpha)$. By construction it is a symplectic form, it equals $\omega$ near $r=0$ and equals $\lambda_{k}\left(\omega+\pi^{*} \sigma\right)$ outside $U$. Since $H_{2}(\bar{X} ; \mathbb{R})$ is generated by $A_{d}$ and $A_{-d}$ and since $a_{d}\left(\hat{\omega}_{k}\right)=a_{d}(\omega)$ and $a_{-d}\left(\hat{\omega}_{k}\right)=a_{-d}(\omega)$, the form $\hat{\omega}_{k}$ is cohomologous to $\omega$. Finally note that the construction can be done to depend smoothly on $k$.

Case: $d<0$ :

Again define $\omega_{k}^{\prime}=\lambda_{k} \omega_{k}$ with

$$
\lambda_{k}=\frac{1+f}{1+k+f} .
$$

Note that $\lambda_{k} \in(0,1]$ and $a_{-d}\left(\omega_{k}^{\prime}\right)=a_{-d}(\omega)$.

Choose $\epsilon$ so that $\{r \leq \epsilon\} \subset U$. Let $\beta(r)$ be a positive increasing function which equals $1+r^{2}$ near $r=0$ and equals $\frac{1+f}{k+1+f}\left(k+1+r^{2}\right)$ near $\epsilon$. To verify that such a function exists, notice that when $k=0$ the expressions $\frac{1+f}{k+1+f}\left(k+1+r^{2}\right)$ and $1+r^{2}$ coincide, and when $k>0$ the expression $1+r^{2}$ is strictly smaller than $\frac{1+f}{k+1+f}\left(k+1+r^{2}\right)$, at least for all $r$ sufficiently small. The function $\beta(r)$ can be constructed by an appropriate smoothing between these two functions.

Define the form $\hat{\omega}_{k}=d(\beta(r) \alpha)$. By construction it is a symplectic form, it equals $\omega$ near $r=0$ and equals $\lambda_{k}\left(\omega+\pi^{*} \sigma\right)$ outside $U$. Since $a_{d}\left(\hat{\omega}_{k}\right)=a_{d}(\omega)$ and $a_{-d}\left(\hat{\omega}_{k}\right)=a_{-d}(\omega)$, the form $\hat{\omega}_{k}$ is cohomologous to $\omega$. Again the construction can be done to depend smoothly on $k$.

Case: $d=0$ :

With $\lambda_{k}=\frac{1}{k+1}$ define

$$
\omega_{k}^{\prime}=\lambda_{k} \omega_{k}=\pi^{*} \sigma+\lambda_{k} d y_{1} \wedge d y_{2} .
$$

Note that $\lambda_{k} \in(0,1]$ and $a_{0}\left(\omega_{k}^{\prime}\right)=a_{0}(\omega)$.

Choose $\epsilon$ so that $\{r \leq \epsilon\} \subset U$. Choose a positive function $\phi(r)$ so that $\phi(r)=1$ near $r=0, \phi(r)=\lambda_{k}$ for $r \geq \epsilon$ and $f\left(\pi^{*} \sigma+\phi(r) d y_{1} \wedge d y_{2}\right)=$ $f(\omega)$. Define $\hat{\omega}_{k}=\pi^{*} \sigma+\phi(r) d y_{1} \wedge d y_{2}$. This is a symplectic form, it equals $\omega$ near $r=0$, equals $\lambda_{k}\left(\omega+k \pi^{*} \sigma\right)$ outside $U$, and is cohomologous to $\omega$. The construction can be done to depend smoothly on $k$.

\section{Topology of spaces of surfaces}

We can now prove the main theorem (see theorem 1.2) if either $d \neq 0$ or $g \neq 0$.

Theorem 8.1. Let $(\bar{X}, \omega)$ be a ruled symplectic 4 -manifold. Fix $d \in \mathbb{Z}$ and suppose that the cohomology assumption $\left(*_{d}\right)$ holds. Let $Z \in \mathcal{S}_{d}(\bar{X})$ be a symplectic surface representing the homology class $A_{d}$. Then the set $\mathcal{S}_{-d}(\bar{X} \backslash Z)$ of symplectic surfaces representing the homology class $A_{-d}$ and disjoint from $Z$ is non-empty and contractible. 
Proof. First we show that $\mathcal{S}_{-d}(\bar{X} \backslash Z)$ is non-empty, i.e. that we can find a symplectic surface $\Sigma \in \mathcal{S}_{-d}$ which is disjoint from $Z$. In the following we will assume that $d \neq 0$ (if $d=0$ then the statement is clearly true by moving $Z$ slightly off itself).

Choose an $\omega$-tame almost complex structure $J$ for which $Z$ is holomorphic. By proposition 4.1 we obtain a $J$-holomorphic foliation $\mathcal{F}$ of $\bar{X}$ by the spheres in the homology class $F$, each of which intersects $Z$ uniquely, transversely and positively. We fix a standard presentation (1) of $\omega$ near $Z$. By lemma 6.1 we can modify the foliation $\mathcal{F}$ in a neighborhood of $Z$ so that it remains symplectic and intersects $Z$ nicely (that is, near $\Sigma$ coincides with the foliation given by the fibers of $\pi$ ).

Choose any smooth section $S$ of $\mathcal{F}$ which is disjoint from $Z$. For $K \geq 0$ sufficiently large, $S$ is $\omega+K \pi^{*} \sigma$-symplectic. By proposition 7.1 there exists a family $\hat{\omega}_{k}$ of symplectic forms which are cohomologous to $\omega$, coincide with $\omega$ near $Z$, and so that $\hat{\omega}_{0}=\omega$ and $S$ is $\hat{\omega}_{K}$-symplectic.

Consider the family $\hat{\omega}_{k}$ of symplectic forms, $k \in[0, K]$. By Moser's method there is a symplectomorphism $\psi:\left(\bar{X}, \hat{\omega}_{0}=\omega\right) \rightarrow\left(\bar{X}, \hat{\omega}_{K}\right)$ which is $i d$ near $Z$. It follows that $\Sigma \stackrel{\text { def }}{=} \psi^{-1}(S)$ is an $\omega$-symplectic surface disjoint from $Z$.

Next we show that $\mathcal{S}_{-d}(\bar{X} \backslash Z)$ is contractible. Consider any $S^{n}$ family of symplectic surfaces $\Sigma_{\lambda} \in \bar{X} \backslash Z$. In the proof of lemma 5.1 we have constructed a family $\mathcal{F}_{\mu}$ of symplectic foliations and a corresponding family $\Sigma_{\mu}$ of sections, so that: $\Sigma_{\mu}=\Sigma_{\lambda}$ is $\omega$-symplectic for $\mu \in S^{n}=$ $\partial B^{n+1}, \Sigma_{\mu}$ is disjoint from $Z$ for all $\mu$, and $\mathcal{F}_{\mu}$ intersects $Z$ uniquely, transversely and positively for all $\mu$. Our goal is to modify the smooth contraction $\Sigma_{\mu}$ in $\bar{X} \backslash Z$ to a symplectic one.

We can assume that none of the surfaces $\Sigma_{\mu}$ intersect a sufficiently small neighborhood of $Z$. Fixing a standard presentation (1) of $\omega$ near $Z$, by the remark following lemma 6.1 we can modify all the foliations $\mathcal{F}_{\mu}$ near $Z$ so that they intersect $Z$ nicely. We denote by $\pi_{\mu}: \bar{X} \rightarrow Z$ the projection along the fibers of $\mathcal{F}_{\mu}$, that is $\pi_{\mu}$ sends each sphere in the foliation $\mathcal{F}_{\mu}$ to its intersection with $Z$.

For each $\mu \in B^{n+1}$ we can choose $K_{\mu}$ sufficiently large so that $\Sigma_{\mu}$ becomes symplectic with respect to the symplectic form $\omega_{\mu}^{\prime} \stackrel{\text { def }}{=} \omega+K_{\mu} \pi_{\mu}^{*} \sigma$. The values for $K_{\mu}$ can be chosen to depend smoothly on $\mu$ and we can assume that $K_{\mu}=0$ for $\mu \in \partial B^{n+1}$ (so that $\omega_{\mu}^{\prime}=\omega$ for $\mu \in \partial B^{n+1}$ ).

Applying proposition 7.1 for each $\mu$ we can find a family $\hat{\omega}_{\mu, k}$ of symplectic forms which are cohomologous to $\omega$, coincide with $\omega$ near $Z$, so that $\hat{\omega}_{\mu, 0}=\omega$ and $\Sigma_{\mu}$ is $\hat{\omega}_{\mu, K_{\mu}}$-symplectic.

For each $\mu \in B^{n+1}$ we can apply Moser's argument to the family $\hat{\omega}_{\mu, k}$ for $k \in\left[0, K_{\mu}\right]$ to obtain a symplectomorphism $\psi_{\mu}:(\bar{X}, \omega) \rightarrow\left(\bar{X}, \hat{\omega}_{\mu, K_{\mu}}\right)$. Note that each $\psi_{\mu}$ is $i d$ near $Z$ and $\psi_{\mu}=i d$ when $\mu \in \partial B^{n+1}$.

The preceding construction can be made to depend smoothly on $\mu$. It follows that $\tilde{\Sigma}_{\mu}=\psi_{\mu}^{-1}\left(\Sigma_{\mu}\right)$ is a family of $\omega$-symplectic surfaces in $\bar{X}$ providing a symplectic contraction of $\Sigma_{\lambda}=\Sigma_{\mu}, \mu \in \partial B^{n+1}$, in $\bar{X} \backslash Z$.

Next consider the only case of theorem 1.2 not covered by the theorem above, i.e. the case $g=d=0$. 
Proposition 8.2. Let $Z \in \mathcal{S}_{0}\left(S^{2} \times S^{2}, \omega\right)$ be a symplectic surface representing the homology class $A_{0}$. Then the set $\mathcal{S}_{0}\left(S^{2} \times S^{2} \backslash Z, \omega\right)$ of symplectic surfaces representing the homology class $A_{0}$ and disjoint from $Z$ is non-empty and contractible.

Proof. First note that $Z \subset S^{2} \times S^{2}$ has a standard symplectic neighborhood of the form $Z \times D^{2}$ with a split symplectic form. Taking a constant section $Z \times\{p t\}$ we see that $\mathcal{S}_{0}\left(S^{2} \times S^{2} \backslash Z, \omega\right)$ is non-empty.

Next, let $\lambda \in S^{n}$ parameterize a family in $S^{2} \times S^{2} \backslash Z$. In other words, let $\Sigma_{\lambda}$ be an $S^{n}$-family of embedded $\omega$-symplectic surfaces homologous to $Z$ and disjoint from $Z$. One can choose a family $J_{\lambda} \subset \mathcal{J}_{Z}$ of almost complex structures on $S^{2} \times S^{2}$ such that $\Sigma_{\lambda}$ is $J_{\lambda}$-holomorphic for each $\lambda$. Since $\mathcal{J}_{Z}$ is contractible, we can extend $J_{\lambda}$ to a $B^{n+1}$-family of almost complex structures in $\mathcal{J}_{Z}$ which coincides with the original family for $\lambda \in \partial B$. According to proposition 4.1 for each $\lambda \in B^{n+1}$ there is a $J_{\lambda^{-}}$ holomorphic foliation of $S^{2} \times S^{2}$ by spheres representing the homology class $A_{0}$. We will denote by $\mathcal{B}_{J_{\lambda}}$ the corresponding foliation of $S^{2} \times S^{2} \backslash Z$ obtained by excluding $Z$. Let's define

$$
Y=\left\{(\lambda, Z) \mid \lambda \in B^{n+1}, Z \in \mathcal{B}_{J_{\lambda}}\right\} .
$$

This is a locally trivial fibration over $B^{n+1}$ (the projection map is given by $(\lambda, Z) \rightarrow \lambda)$ with the fiber homeomorphic to $D^{2}$. Thus we can extend the section $\Sigma_{\lambda}$ defined for $\lambda \in \partial B^{n+1}$ to a global section of $Y$. This gives the required symplectic isotopy of $\Sigma_{\lambda}$ to a constant family.

The local isotopy claimed in the introduction (see theorem 1.1) is now an easy consequence.

Corollary 8.3. Let $(M, \omega)$ be a symplectic 4-manifold and let $\Sigma \subset M$ be an embedded symplectic surface. Then there is a (arbitrarily small) neighborhood $U$ of $\Sigma$ so that the set $\mathcal{S}(U, \omega)$ of embedded symplectic surfaces in $U$ homologous to $\Sigma$ is weakly contractible.

Proof. Let $U$ be any open standard symplectic neighborhood of $\Sigma$, i.e. a multiple $c \omega$ of $\omega$ is given by the standard form (11). Let $\Sigma_{\lambda}$ be any $S^{n}$ family in $\mathcal{S}(U, \omega)$. As described in section 2 we can compactify $(U, c \omega)$ to a standard symplectic sphere bundle $\bar{X}$. In particular $U$ is symplectomorphic to $\bar{X} \backslash \Sigma_{\infty}$ for a symplectic surface $\Sigma_{\infty} \in \bar{X}$. By theorem $8.1 \Sigma_{\lambda}$ can be symplectically contracted in $\bar{X} \backslash \Sigma_{\infty}$ and hence in $U$.

The proof of the main theorem 8.1 can be easily generalized as follows:

Proposition 8.4. Let $(\bar{X}, \omega)$ be a ruled symplectic 4-manifold. Fix $d \neq 0$ and suppose that the cohomology assumption $\left(*_{d}\right)$ holds. Let $Z_{\lambda} \in \mathcal{S}_{d}(\bar{X})$, $\lambda \in S^{n}$, be a family of symplectic surfaces representing the homology class $A_{d}$. Then there exists a corresponding family $\Sigma_{\lambda}$ of symplectic surfaces representing the homology class $A_{-d}$ so that for each $\lambda \in S^{n}$ the surface $\Sigma_{\lambda}$ is disjoint from $Z_{\lambda}$. Moreover, the set of such families $\left\{\Sigma_{\lambda}\right\}$ is contractible.

Corollary 8.5. Let $(\bar{X}, \omega)$ be a ruled symplectic 4-manifold satisfying the cohomology assumption $\left(*_{d}\right)$. Then $\mathcal{S}_{d}(\bar{X}, \omega)$ is homotopy equivalent to $\mathcal{S}_{-d}(\bar{X}, \omega)$. 
Proof. Consider the set $Y$ of pairs $(Z, \Sigma)$ with $Z \in \mathcal{S}_{d}, \Sigma \in \mathcal{S}_{-d}$, and $Z$ and $\Sigma$ disjoint. The projection maps $Y \rightarrow \mathcal{S}_{d}$ and $Y \rightarrow \mathcal{S}_{-d}$ are fibrations with contractible fiber and hence are both homotopy equivalences.

\section{Relations with groups of symplectomor- phisms}

Motivated by [3, we consider some obvious connections between the groups of symplectomorphisms of a ruled symplectic 4-manifold and the sets of symplectic surfaces. Let $(\bar{X}, \omega)$ be a ruled symplectic 4-manifold. Fix $d \neq 0$ and suppose that $(\bar{X}, \omega)$ satisfies the homology assumption $\left(*_{d}\right)$.

First we introduce some notations. We denote by $\operatorname{Symp}^{h}(\bar{X}, \omega)$ the group of symplectomorphisms of $\bar{X}$ which act as identity on homology. Given $Z \in \mathcal{S}_{d}(\bar{X})$, we denote by $\operatorname{Symp}_{[Z]}^{h}(\bar{X}, \omega) \subset \operatorname{Symp}_{\bar{Z}}^{h}(\bar{X}, \omega) \subset \operatorname{Symp}_{Z}^{h}(\bar{X}, \omega) \subset$ $\operatorname{Symp}^{h}(\bar{X}, \omega)$ the subsets of $\operatorname{Symp}^{h}(\bar{X}, \omega)$ consisting of symplectomorphisms which, respectively, fix a neighborhood of $Z$, fix $Z$, and preserve $Z$.

Consider the action of $\operatorname{Symp}_{[Z]}^{h}(\bar{X}, \omega)$ on $S_{-d}(\bar{X} \backslash Z)$. By theorem 8.1 any two symplectic surfaces in $S_{-d}(\bar{X} \backslash Z)$ can be joined by a path of such symplectic surfaces. None of these surfaces intersect a sufficiently small neighborhood of $Z$ and so this path can be generated by a Hamiltonian which is identity in a neighborhood of $Z$. It follows that the action above is transitive. By the same theorem, the set $S_{-d}(\bar{X} \backslash Z)$ is in fact contractible. The stabilizer of a surface $\Sigma \in S_{-d}(\bar{X} \backslash Z)$ is the set of symplectomorphisms of $\bar{X}$ which preserve $\Sigma$ and fix a neighborhood of $Z$. As shown in [3, this set is also contractible. It follows that

Lemma 9.1. Assuming the cohomology assumption $\left(*_{d}\right)$, the group of symplectomorphisms of $(\bar{X}, \omega)$ which act as identity on homology and fix a neighborhood of a symplectic surface $Z \in \mathcal{S}_{d}(\bar{X})$ is contractible.

Next consider the action of $\operatorname{Symp}_{Z}^{h}(\bar{X}, \omega)$ on $S_{-d}(\bar{X} \backslash Z)$. As before this action is transitive and the set $S_{-d}(\bar{X} \backslash Z)$ is contractible. The stabilizer of a surface $\Sigma \in S_{-d}(\bar{X} \backslash Z)$ consists of symplectomorphisms which preserve both $\Sigma$ and $Z$. By [3], this set is homotopy equivalent to the set $D_{0, \infty}$ of fiber preserving diffeomorphisms of a standard sphere bundle of degree $d$ over $\Sigma$ which also preserve the sections $\Sigma_{0}$ and $\Sigma_{\infty}$. It follows that $\operatorname{Symp}_{Z}^{h}(\bar{X}, \omega)$ is homotopy equivalent to $D_{0, \infty}$.

By 3], given any two pairs of non-intersecting symplectic sections $\left(Z_{1}, \bar{Z}_{1}\right),\left(Z_{2}, \bar{Z}_{2}\right)$ with $Z_{1}, Z_{2} \in \mathcal{S}_{d}(\bar{X})$ and $\bar{Z}_{1}, \bar{Z}_{2} \in \mathcal{S}_{-d}(\bar{X})$, there exists a symplectomorphism in $\operatorname{Symp}^{h}(\bar{X}, \omega)$ mapping $Z_{1}$ to $Z_{2}$ and mapping $\bar{Z}_{1}$ to $\bar{Z}_{2}$. In particular, the group $\operatorname{Symp}^{h}(\bar{X}, \omega)$ acts transitively on $\mathcal{S}_{d}(\bar{X})$. The stabilizer of this action corresponding to $Z \in S_{d}(\bar{X})$ is precisely the set $\operatorname{Symp}_{Z}^{h}(\bar{X}, \omega)$ of symplectomorphisms which preserve $Z$ considered before. Therefore we obtain the following:

Lemma 9.2. Assuming the cohomology assumption $\left(*_{d}\right)$, the group $\operatorname{Symp}^{h}(\bar{X}, \omega)$ acts transitively on the set $\mathcal{S}_{d}(\bar{X}, \omega)$ with the stabilizer homotopy equivalent to $D_{0, \infty}$. 
It is interesting to note the connection with the theorem of [1] (see also 11]) which can be formulated as follows. Let $\bar{X}=\Sigma \times S^{2}$ and let $\omega_{\mu}=\mu \sigma_{\Sigma}+\sigma_{S^{2}}$. Denote by $\operatorname{Symp}_{i d}\left(\bar{X}, \omega_{\mu}\right) \subset \operatorname{Symp}^{h}\left(\bar{X}, \omega_{\mu}\right)$ the group of symplectomorphisms which are isotopic to $i d$ as diffeomorphisms, and denote by $D$ the identity component of the group of fiber preserving diffeomorphisms of $\Sigma \times S^{2}$. The theorem states that as $\mu$ tends to infinity, the groups $\operatorname{Symp}_{i d}\left(\bar{X}, \omega_{\mu}\right)$ tend to a limit which is homotopy equivalent to $D$.

\section{References}

[1] M. Abreu and D. McDuff. Topology of symplectomorphism groups of rational ruled surfaces. J. Amer. Math. Soc., 13(4):971-1009 (electronic), 2000.

[2] P. Biran. Lagrangian barriers and symplectic embeddings. Geom. Funct. Anal., 11(3):407-464, 2001.

[3] J. Coffey. Symplectomorphism groups and isotropic skeletons. Geom. Topol., 9:935-970 (electronic), 2005.

[4] R. Fintushel and R. J. Stern. Symplectic surfaces in a fixed homology class. J. Differential Geom., 52(2):203-222, 1999.

[5] M. Gromov. Pseudoholomorphic curves in symplectic manifolds. Invent. Math., 82(2):307-347, 1985.

[6] R. Hind and A. Ivrii. Isotopies of high genus lagrangian surfaces. In preparation.

[7] H. Hofer, V. Lizan, and J.-C. Sikorav. On genericity for holomorphic curves in four-dimensional almost-complex manifolds. J. Geom. Anal., 7(1):149-159, 1997.

[8] F. Lalonde and D. McDuff. The classification of ruled symplectic 4-manifolds. Math. Res. Lett., 3(6):769-778, 1996.

[9] F. Lalonde and D. McDuff. J-curves and the classification of rational and ruled symplectic 4-manifolds. In Contact and symplectic geometry (Cambridge, 1994), volume 8 of Publ. Newton Inst., pages 3-42. Cambridge Univ. Press, Cambridge, 1996.

[10] D. McDuff. From symplectic deformation to isotopy. In Topics in symplectic 4-manifolds (Irvine, CA, 1996), First Int. Press Lect. Ser., I, pages 85-99. Internat. Press, Cambridge, MA, 1998.

[11] D. McDuff. Symplectomorphism groups and almost complex structures. In Essays on geometry and related topics, Vol. 1, 2, volume 38 of Monogr. Enseign. Math., pages 527-556. Enseignement Math., Geneva, 2001.

[12] D. McDuff and D. Salamon. J-holomorphic curves and quantum cohomology, volume 6 of University Lecture Series. American Mathematical Society, Providence, RI, 1994.

[13] V. Shevchishin. Pseudoholomorphic curves and the symplectic isotopy problem. Preprint, math.SG/0010262. 
[14] B. Siebert and G. Tian. On the holomorphicity of genus two Lefschetz fibrations. Ann. of Math. (2), 161(2):959-1020, 2005.

Richard Hind

Department of Mathematics

University of Notre Dame

Notre Dame, IN 46556

email: hind.1@nd.edu

Alexander Ivrii

Départment de Mathématiques et de Statistique

Université de Montréal

CP 6128 succ Centre-Ville

Montréal, QC H3C 3J7, Canada

email: ivrii@DMS.UMontreal.CA 